\title{
Synthesis of Silver Nanoparticles Using Flavonoid Hesperidin and Pharmacological Evaluation of its Free Radical Scavenging Activity
}

\author{
Anish Stephen', Mahesh Kumar D²*
}

\begin{abstract}
${ }^{1}$ Assistant Professor, Department of Pharmacology, PK Das Institute of Medical sciences, Vaniamkulam, Ottapalam, Kerala, India.

Email: anishstephen@gmail.com Orcid ID: 0000-0002-7941-8754

2Associate Professor, Department of Pharmacology, PK Das Institute of Medical sciences, Vaniamkulam, Ottapalam, Kerala, India. Email: saakethbhagat@gmail.com

Orcid ID: 0000-0002-0535-601X
\end{abstract}

*Corresponding author

Received: 17 September 2021

Revised: 07 November 2021

Accepted: 18 November 2021

Published: 22 December 2021

\begin{abstract}
Background: Silver Nanoparticles are drawing significant attention from the scientific community to explore a wide range of its medical applications. Human body is under constant stress due to free radicals generated by the physiological and pathological conditions in the body. Scavenging systems or Antioxidants can help alleviate the damages caused by these radicals which can influence the course of progress in several chronic diseases with an inflammatory background. External antioxidants supplement and facilitate the overwhelmed scavenging systems in the body. Silver Nanoparticles can enhance the therapeutic effects of phytochemicals. Aim: To Synthesize silver nanoparticles using the phytochemical Hesperidin and studying its Free radical scavenging activity. Methods: Silver Nanoparticles are synthesized using chemical reduction method. The synthesis is confirmed using spectrophotometric studies. Free Radical scavenging activity is detected using 1, 1-diphenyl-2-picrylhydrazyl (DPPH •) free radical scavenging assay. Results: Silver nanoparticles were successfully synthesized which was confirmed by the change in color of the solution and peak absorbance peak at $420 \mathrm{nM}$ on spectrophotometric studies.Hesperidin Silver Nanoparticles exhibited higher free radical scavenging activity when compared with pure hesperidin and standard Ascorbic acid. Conclusion: Hesperidin can ideally be used for the synthesis of silver nanoparticles and the synthesized Silver Nanoparticles enhances the free radical scavenging activity of Hesperidin which can further be evaluated by In Vivo studies.
\end{abstract}

Keywords:- Silver Nanoparticles, Hesperidin, Free Radical Scavenging activity.

\section{INTRODUCTION}

Nanoparticles have been in the research limelight for over the past several years with a tremendous momentum with which obscurities related to it are being uncovered. It has multidisciplinary utilities and because of which research on nanoparticles is spread over various disciplines like Medical sciences, Engineering sciences, Physical and Materialsciences, etc.

Nanoparticles are particles with any of its cross sectional length between $1 \mathrm{~nm}$ to $100 \mathrm{~nm}$. The properties exhibited by the metallic nanoparticles are size and shape-dependent.[1] Silver nanoparticles have been known since the times when ancient medical texts were written. 
Annals of International Medical and Dental Research

E-ISSN: 2395-2822 | P-ISSN: 2395-2814

Vol-8, Issue-1 | January-February 2022

DOI: $10.53339 /$ aimdr.2022.8.1.26

Page no- 192-199 | Section- Research Article (Pharmacology)

Whole new perspective of silver nanoparticles has come up once again in recent years, which includes its synthesis using plant extracts which is considered to be an environmentally benign process. Silver nanoparticles exhibit a variety of therapeutic effects and one among them is their anti-inflammatory effect.[2] Inflammation is the basis of pathogenesis of various chronic human diseases. It is also implicated as an etiology in various diseases. Acute inflammation is a lifesaving aid to curb infections and infestations but if proceeds beyond the framework of acute inflammation the constituent cells in the inflammation change and lead to two processes running simultaneously, one which leads to tissue damage and the other one leading to deformation and fibrosis. [3] Herbal sources can be considered at the grass root level from which the majority of the Anti Inflammatory armamentarium grew up. Herbs are rich in phytochemicals and it is these phytochemicals that are responsible for the anti-inflammatory and other therapeutic properties. At present, from the modern medicine perspective, corticosteroids,non-steroidal anti-inflammatory drugs, immunosuppressant, histamine receptor antagonists, etc. are the constituents of the antiinflammatory therapeutic armamentarium. In spite of their documented efficacy, most of them have deleterious side effects on the body. Analogous to the fact that inflammation can produce both deleterious and advantageous effects on the body; drugs affecting the inflammatory processes also have contradictory Effects. Due to these reasons, drug exploration in plants was sought after and those involved a multidisciplinary approach combining modern and traditional medicine practices towards the single aim of drug development.[4] Since phytotherapeutics were more dependent on crude plant extracts, they were prone to lower patient compliance. This could be attributed to side effects or lack of efficacy which may be due to the lacunae in delivery of required constituents in the required concentration or maybe due to the presence of other constituents in the extract mixture which can antagonize the beneficial effects and may also exert side effects. Hence, the need for a scientific approach to deliver the components in a sustained manner to increase patient compliance and optimization of pharmacokinetics and pharmacodynamic parameters are raised.[5] An ideal scenario of the confluence of phytopharmacology and nanotechnology is the synthesis of silver nanoparticles using plant extracts which is considered as an environmentally benign and eco friendly process. It is cited in various research literature that silver nanoparticle formation is due to the reduction of silver nitrate by the phytochemical rich plant extracts. These plant extracts form a capping over the synthesized silver nanoparticles, which stabilizes the silver nanoparticles by preventing its agglomeration and also this capping bestows additional pharmacological properties on the newly synthesized silver nanoparticles. [6] This capping also helps in delivering the active compounds present in the extracts to the body. It is based on this principle that this study focuses on synthesis of silver nanoparticles directly using a purified phytochemical which in this study is a flavonoid Hesperidin rather than using a plant extract and evaluating this synthesized silver nanoparticles for its free radical scavenging activity. Hesperidin belongs to the phytochemical class Flavonoids and subclass flavanone glycoside and is found in citrus fruits. Hesperidin is abundant in citrus fruits. Hesperidin has been scientifically 
Annals of International Medical and Dental Research

E-ISSN: 2395-2822 | P-ISSN: 2395-2814

Vol-8, Issue-1 | January-February 2022

DOI: $10.53339 /$ aimdr.2022.8.1.26

Page no- 192-199 | Section- Research Article (Pharmacology)

studied and is shown to exhibit significant antiinflammatory properties which can be compared with NSAIDs - Indomethacin. [7] By using pure Hesperidin for synthesis of silver nanoparticles, capping of silver nanoparticles with Hesperidin will occur exclusively since there is no other compound in the reaction mixture to intervene in this process. There is a bulk of research activity pertaining to the synthesis of silver nanoparticles using plant extracts but, negligible with pure phytochemicals. This study aims to explore the utility of flavonoids (Hesperidin) for the synthesis of silver nanoparticles and evaluation of its potential as a free radical scavenger (antioxidant).

\section{MATERIAL AND METHODS}

\section{Synthesis of silver nanoparticles using phytochemical hesperidin}

Materials:Flavonoid Hesperidin (HPLC 90\%) was purchased from Xian Xiaocao Botanical Development Co., Ltd., China. Silver nitrate (99.9999\% trace metals basis) was purchased from Sigma Aldrich. 99.8\% methanol was purchased from ILE Chennai.Milli-Q system was used for deionized water. Silver nanoparticles were prepared by chemical reduction of salt solution of silver nitrate using the flavonoid Hesperidin. Hesperidin solution was prepared by using Methanol $(99.8 \%)$ as a solvent in the strength $300 \mu \mathrm{g} / \mathrm{mL}$. The $\mathrm{pH}$ of the Hesperidin solution which is acidic $(\mathrm{pH}$ around 3.2) is raised to a basic level of around 12 by adding drops of freshly prepared $\mathrm{NaOH}$. Aqueous silver nitrate solution was prepared in the strength of $1 \mathrm{mM}$ by mixing $169.8 \mathrm{mg}$ of silver nitrate in glass beakers having 1 liter of deionized water (AgNO3 molecular weight is
169.87 Da) . Ninety $\mathrm{ml}$ of aqueous solution of silver nitrate was taken in a flask and kept on a magnetic stirrer into which $10 \mathrm{ml}$ of Hesperidin solution was added drop by drop using a micropipette.After adding Hesperidin solution into the aqueous solution of silver nitrate, the reaction mixture was observed for change in colour. Change in colour signifies the formation of silver nanoparticles. The colour of the solution will become dark yellow or golden colour. Once silver nanoparticle formation is confirmed, the solution should be heated at around $70^{\circ} \mathrm{C}$ so that methanol can evaporate out of the reaction mixture since the boiling point of methanol is $64.7^{\circ} \mathrm{C}$. The remaining reaction mixture is left to dry on watch glass at room temperature. After drying, the residual silver nanoparticles are left behind in the powdered form which can be scrapped carefully into a small container which can be used for further studies.

\section{Characterization of the silver nanoparticles synthesized using hesperidin}

The purpose of characterization is to confirm the formation of silver nanoparticles and further study its size, structure and the nature of capping on it. Synthesized silver nanoparticles were subjected to Spectrophotometric studies to determine the confirmation of synthesis of silver nanoparticles in colloid solution and qualitative estimation of the colloid solution.

\section{Evaluation of free radical scavenging activity}

a) Determination of free radical scavenging activity using 1, 1-diphenyl-2-picrylhydrazyl $(\mathrm{DPPH} \bullet$ ) free radical scavenging assay.DPPH 
Annals of International Medical and Dental Research E-ISSN: 2395-2822 | P-ISSN: 2395-2814 Vol-8, Issue-1 | January-February 2022 DOI: $10.53339 /$ aimdr.2022.8.1.26 Page no- 192-199 | Section- Research Article (Pharmacology)

free radical scavenging assay was done with the method of Yohozowa et al.8] Following concentrations of Hesperidin silver nanoparticles in double distilled water (500 $\mu \mathrm{g} / \mathrm{ml}, 250 \mu \mathrm{g} / \mathrm{ml}, 125 \mu \mathrm{g} / \mathrm{ml}, 62.5 \mu \mathrm{g} / \mathrm{ml}$, $31.25 \mu \mathrm{g} / \mathrm{ml}, 15.625 \mu \mathrm{g} / \mathrm{ml}$ ) were Incubated. The same process was repeated for pure Hesperidin. Control for Silver nanoparticles and ascorbic acid was taken as $1.9 \mathrm{ml}$ of DPPH solution $(80 \mu \mathrm{g} / \mathrm{ml})$ with $0.1 \mathrm{ml}$ of double distilled water and for Hesperidin was taken as $1.9 \mathrm{ml}$ of DPPH solution $(80 \mu \mathrm{g} / \mathrm{ml})$ with $0.1 \mathrm{ml}$ of methanol. Ascorbic acid was taken in the same concentrations. The resultant absorbance was recorded at $517 \mathrm{~nm}$ using ELICO Double beam-SL210 UV-VIS Spectrophotometer. The DPPH free radical scavenging activity was calculated using the following formula:

$\%$ scavenging $=$

Absorbance of control -Absorbance of test sample $\times 100$

\section{Absorbance of control}

All the readings were taken in triplicate and their mean value was taken in consideration. Results were expressed as Mean \pm Standard Error of means (SEM). Statistical analysis will be performed using GraphPad Instat software. Linear regression, Test of linearity (expressed in $P$ value), Interpolation of the standard curve to get the IC50 value will be done.

\section{RESULTS}

\section{- Synthesis of silver nanoparticles}

Hesperidin solution on adding with aqueous solution of silver nitrate brings in a change in colour of the reaction mixture from colourless to dark brown. The change in colour is evident after adding a few drops of Hesperidin. Change in colour in the solution infers the formation of silver nanoparticles by reduction of the aqueous solution of silver nitrate on exposure to Hesperidin solution. There was no change in the control mixture in which there was no Hesperidin but only $10 \mathrm{ml}$ of methanol.

\section{- Characterisation of silver nanoparticles synthesized using hesperidin}

Spectrophotometric analysis of the synthesized silver nanoparticles

The sample was taken after complete mixture of $10 \mathrm{ml}$ of Hesperidin soon after the color of the reaction mixture changed completely to brownish yellow, that is, around 2 mins after the last drop of Hesperidin solution was added. Maximum OD was observed at a wavelength of $420 \mathrm{~nm}$. This signifies that silver nanoparticles are formed in the solution and that silver ions are reduced to metallic silver.

\section{- In vitro evaluation of free radical scavenging activity}

a. Determination of free radical scavenging activity using $\mathrm{DPPH} \bullet$ free radical scavenging assay

After incubating the reaction mixture for 30 mins in dark, the resultant absorbance was recorded at $517 \mathrm{~nm}$ using ELICO Double beamSL210 UV-VIS Spectrophotometer.

$\%$ Inhibition was calculated using the formula:

$\%$ scavenging $=$

Absorbance of control -Absorbance of test sample $x 100$

Absorbance of control 
Annals of International Medical and Dental Research

E-ISSN: 2395-2822 | P-ISSN: 2395-2814

Vol-8, Issue-1 | January-February 2022

DOI: $10.53339 /$ aimdr.2022.8.1.26

Page no- 192-199 | Section- Research Article (Pharmacology)

Results are expressed as Mean \pm Standard Error

of mean (SEM).

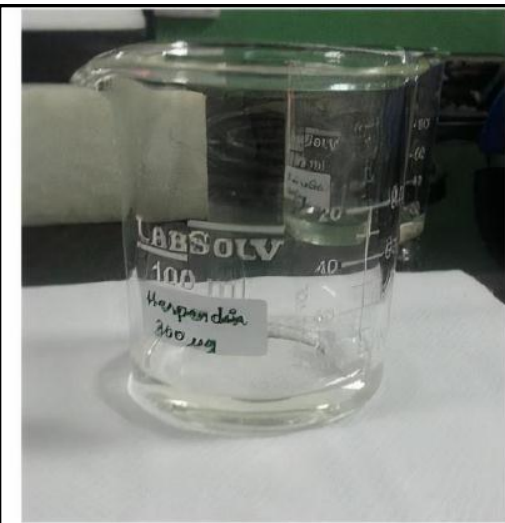

Hesperidin solution

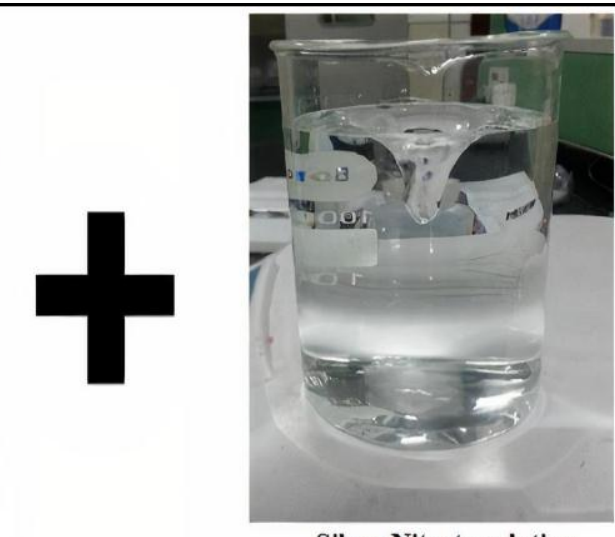

Silver Nitrate solution

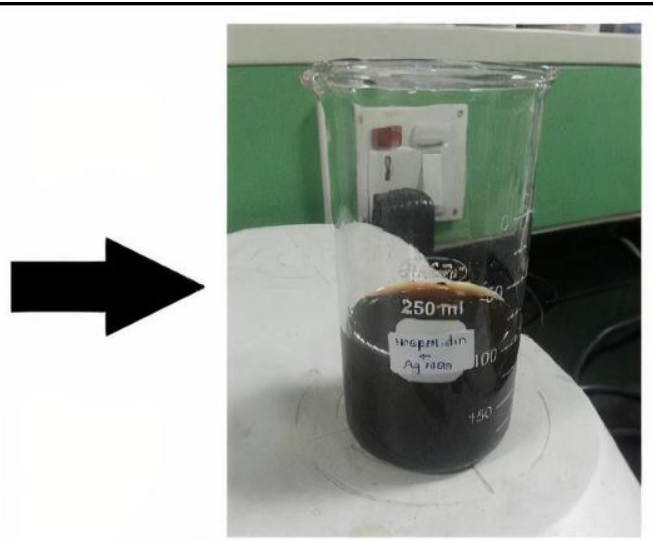

Silver Nitrate $(90 \mathrm{ml})+$ Hesperidin $(10 \mathrm{ml})$

Figure 1: Change in color of the mixture signifying formation of silver nanoparticles.

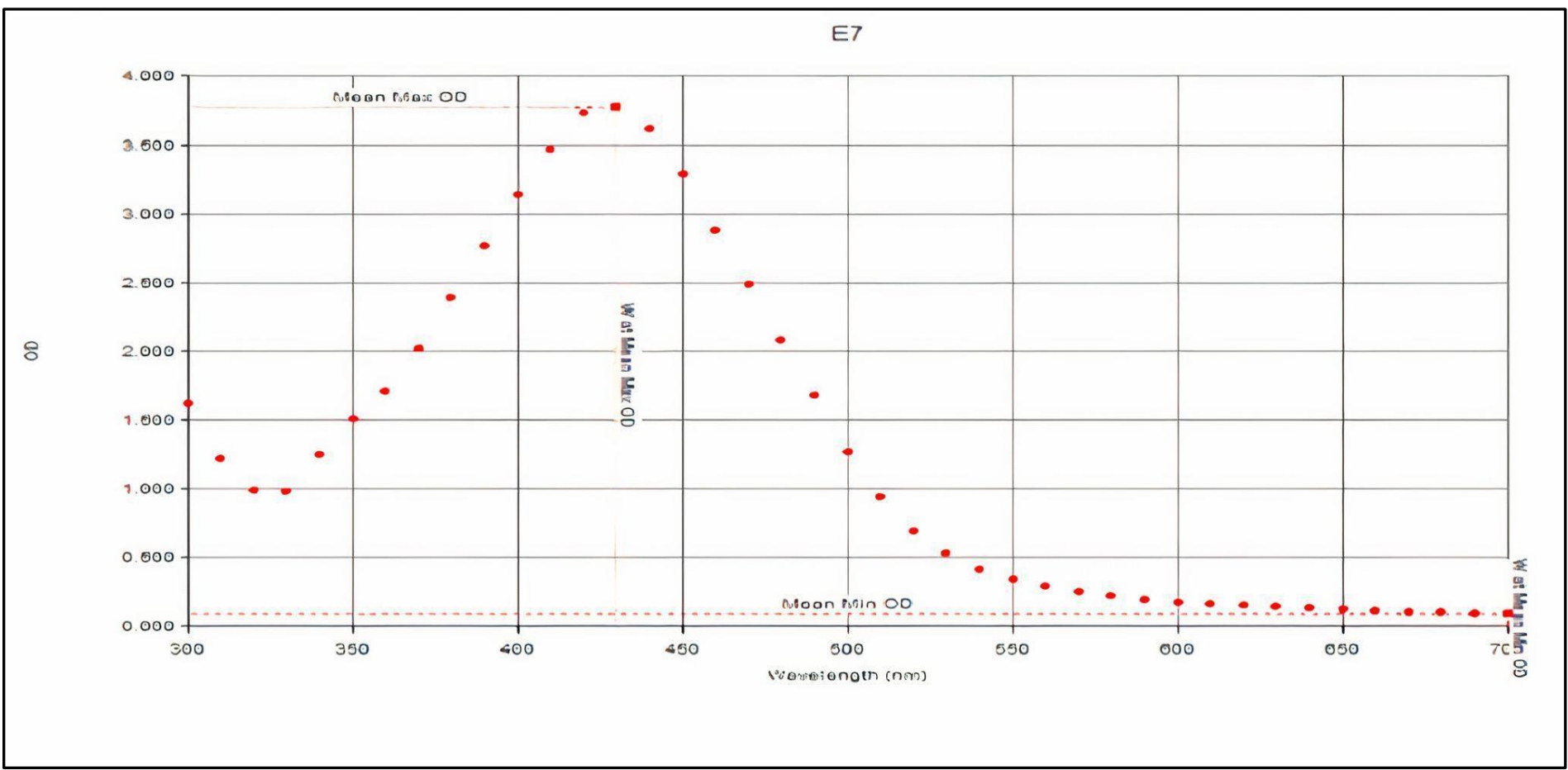

Figure 2: Spectrophotometric reading showing peak absorbance at $420 \mathrm{~nm}$. 
Annals of International Medical and Dental Research E-ISSN: 2395-2822 | P-ISSN: 2395-2814

Vol-8, Issue-1 | January-February 2022 DOI: 10.53339/aimdr.2022.8.1.26

Page no- 192-199 | Section- Research Article (Pharmacology)

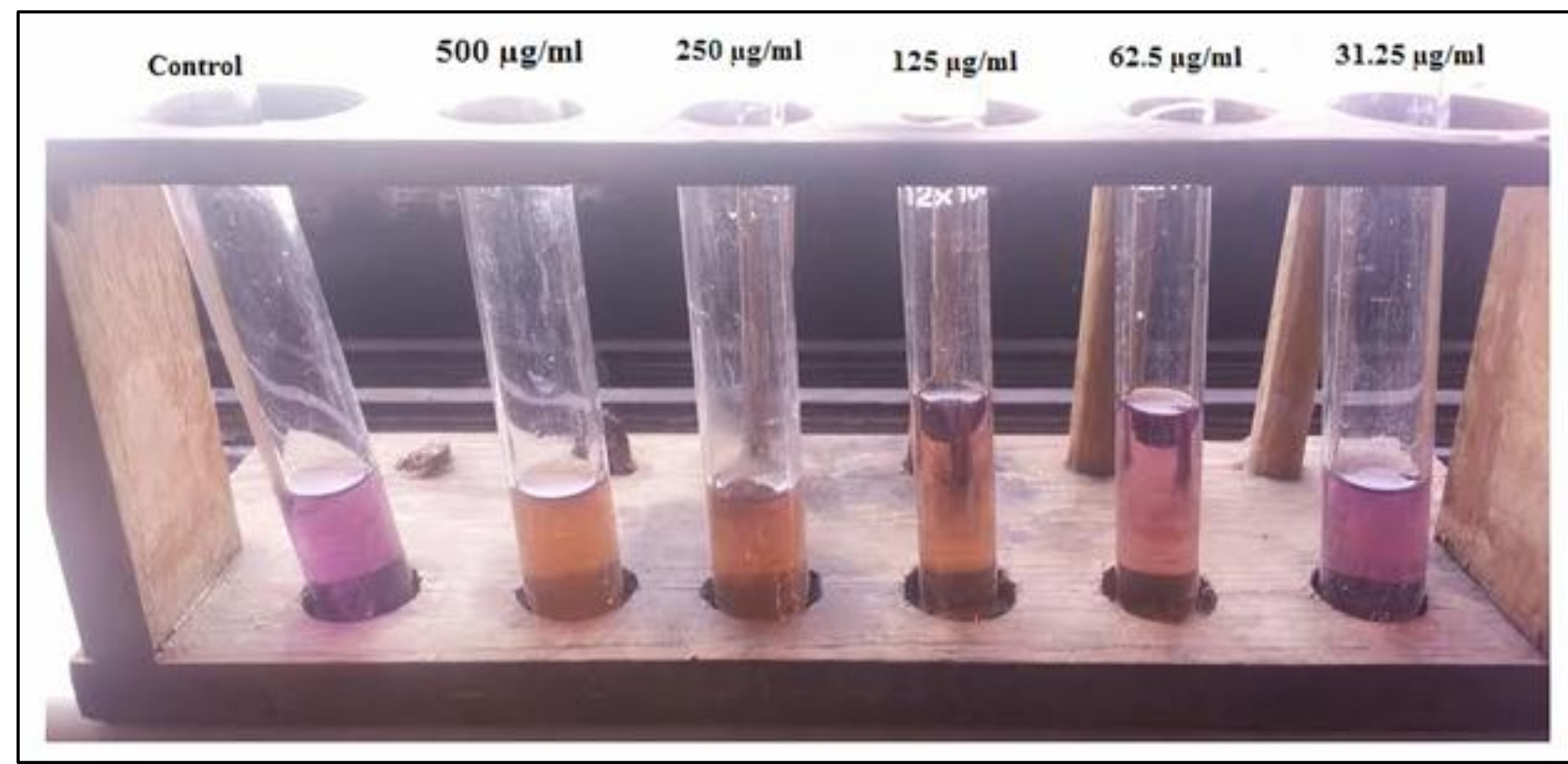

Figure 3: DPPH• free radical scavenging activity at various concentrations

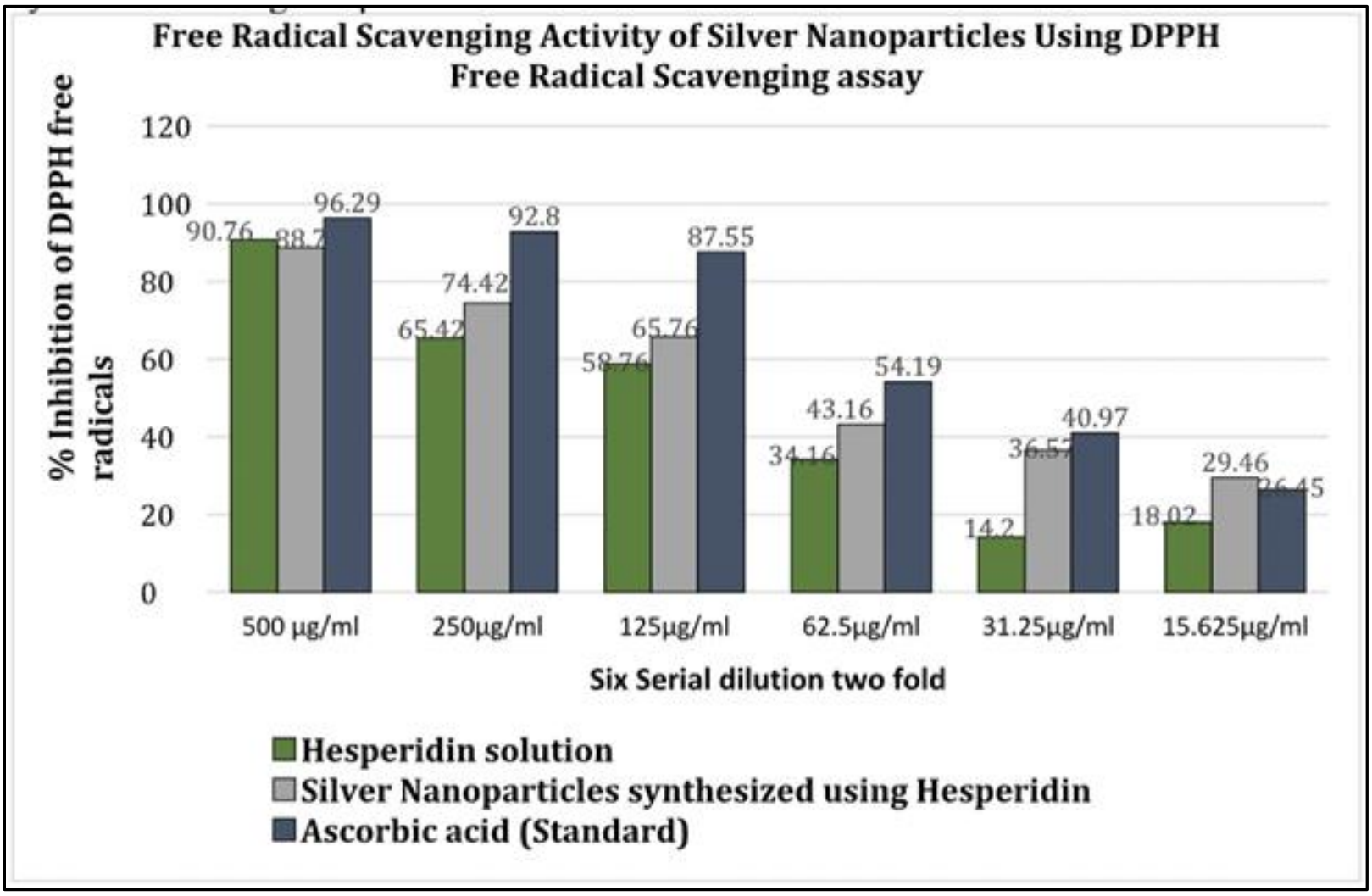

Figure 4: \% Inhibition of DPPH Radicals by Ascorbic acid, Hesperidin \&; Hesperidin silver Nanoparticles. 
Annals of International Medical and Dental Research

E-ISSN: 2395-2822 | P-ISSN: 2395-2814

Vol-8, Issue-1 | January-February 2022

DOI: $10.53339 /$ aimdr.2022.8.1.26

Page no- 192-199 | Section- Research Article (Pharmacology)

\section{DISCUSSION}

Inflammation in several scenarios tends to act as a double edged sword with deleterious consequences on the body of the host. Chronic inflammation is the basis of a bulk of diseases involving human beings, few examples being diabetes mellitus, rheumatoid arthritis, acute coronary syndrome, cerebrovascular diseases and various malignancies. In this study, we report the synthesis of silver nanoparticles using the phytochemical Hesperidin by an environmentally benign procedure and evaluation of its free radical scavenging activity. Formation of silver nanoparticles was confirmed by physical observation which revealed a change of colour of the reaction mixture into golden yellow colour. Silver Nanoparticles exhibit a yellowish-brown colour in aqueous solution due to excitation of surface plasmon vibrations.[9] UV-VIS spectroscopy data exhibited a sharp peak at 420 $\mathrm{nM}$ wavelength and an optical density of 3.78 .

Reactive oxygen species (ROS) are inextricably linked to malignant diseases, diabetes, atherosclerosis, chronic inflammation, ischemia-reperfusion injury etc., and are produced continuously in most tissues.[10] Superoxide and hydroxyl radicals,hydrogen peroxide, singlet oxygen are known to be cytotoxic and have been implicated in the etiology and pathogenesis of several chronic diseases.[11] Oxygen derived radicals represent the most important class of radical species generated in living systems.[12] The harmful effect of free radicals causing potential biological damage is oxidative stress. Epidemiological and in vitro studies have revealed that medicinal plant extracts protects biological systems from oxidative stress.[13]
In this study silver nanoparticles synthesized using Hesperidin was evaluated for its free radical scavenging activity along with pure Hesperidin and Ascorbic acid (standard). Silver nanoparticles exhibit free radical scavenging activity at par with pure Hesperidin and the standard Ascorbic acid. It can be seen that silver nanoparticles synthesized using Hesperidin have better activity compared to that of Hesperidin at lower concentrations. The free radical scavenging activity of silver nanoparticles synthesized using Hesperidin can be attributed to the capping of silver nanoparticles with Hesperidin. DPPH is nonphysiological, stable radical and most widely used in assay for screening antioxidant activity of plant extracts. [14] DPPH is reduced to diphenylpicryl hydrazine by products having antioxidant properties in a concentrationdependent manner. The observed IC50 values were $20.255 \mu \mathrm{g} / \mathrm{ml}$ for Ascorbic acid, 36.778 $\mu \mathrm{g} / \mathrm{ml}$ for pure Hesperidin and $34.714 \mu \mathrm{g} / \mathrm{ml}$ for silver nanoparticles synthesized using Hesperidin. It was observed that silver nanoparticles synthesized using Hesperidin have better activity than pure Hesperidin and also than the standard ascorbic acid at concentrations of $25 \mu \mathrm{g} / \mathrm{ml}$ and lower.

\section{CONCLUSIONS}

In this research study Silver nanoparticles were synthesized using the phytochemical Hesperidin which is a novel eco-friendly method. The synthesized silver nanoparticles were evaluated for its free radical scavenging activity using in vitro assays like DPPH • free radical scavenging assay. It was found that silver nanoparticles exhibited better free radical scavenging activity than pure hesperidin and its 
Annals of International Medical and Dental Research

E-ISSN: 2395-2822 | P-ISSN: 2395-2814

Vol-8, Issue-1 | January-February 2022

DOI: 10.53339/aimdr.2022.8.1.26

Page no- 192-199 | Section- Research Article (Pharmacology)

strong activity at low concentrations were worth mentioning.

\section{REFERENCES}

1. Choi $\mathrm{Y}$, Ho NH, Tung CH. Sensing phosphatase activity by using gold nanoparticles. Angew Chem Int Ed Engl. 2007;46(5):707-9. doi: 10.1002/anie.200603735.

2. Wong KK, Cheung SO, Huang L, Niu J, Tao C, Ho $\mathrm{CM}$, et al. Further evidence of the anti-inflammatory effects of silver nanoparticles. ChemMedChem. 2009;4(7):1129-35. doi: 10.1002/cmdc.200900049.

3. Wells RG. Tissue mechanics and fibrosis. Biochim Biophys Acta. 2013;1832(7):884-890. doi:10.1016/j.bbadis.2013.02.007

4. Bellik Y, Hammoudi SM, Abdellah F, Iguer-Ouada M, Boukraâ L. Phytochemicals to prevent inflammation and allergy. Recent Pat Inflamm Allergy Drug Discov. 2012;6(2):147-58. doi: 10.2174/187221312800166886.

5. Bonifácio BV, Silva PB, Ramos MA, Negri KM, Bauab TM, Chorilli M. Nanotechnology-based drug delivery systems and herbal medicines: a review. Int J Nanomedicine. 2014;9:1-15. doi:10.2147/IJN.S52634

6. Fayaz AM, Balaji K, Girilal M, Yadav R, Kalaichelvan PT, Venketesan R. Biogenic synthesis of silver nanoparticles and their synergistic effect with antibiotics: a study against gram-positive and gramnegative bacteria. Nanomedicine. 2010;6(1):103-109.

7. Garg A, Garg S, Zaneveld LJ, Singla AK. Chemistry and pharmacology of the Citrus bioflavonoid hesperidin. Phytother Res. 2001;15(8):655-69. doi: 10.1002/ptr.1074.

8. Yokozawa T, Chen CP, Dong E, Tanaka T, Nonaka GI, Nishioka I. Study on the inhibitory effect of tannins and flavonoids against the 1,1-diphenyl-2 picrylhydrazyl radical. Biochem Pharmacol. 1998;56(2):213-22. doi: 10.1016/s0006-2952(98)001282.

9. Song JY, Kim BS. Rapid biological synthesis of silver nanoparticles using plant leaf extracts. Bioprocess Biosyst Eng. 2009;32(1):79-84. doi: 10.1007/s00449008-0224-6.

10. Dröge W. Oxidative stress and ageing: is ageing a cysteine deficiency syndrome? Philos Trans R Soc Lond B Biol Sci. 2005;360(1464):2355-2372. doi:10.1098/rstb.2005.1770

11. Bergamini CM, Gambetti S, Dondi A, Cervellati C. Oxygen, reactive oxygen species and tissue damage. Curr Pharm Des. 2004;10(14):1611-26. doi: 10.2174/1381612043384664.

12. Miller DM, Buettner GR, Aust SD. Transition metals as catalysts of "autoxidation" reactions. Free Radic Biol Med. 1990;8(1):95-108. doi: 10.1016/08915849(90)90148-c.

13. Chaudhary SR, Chavan MJ, Gaud RS. Antiinflammatory and Analgesic activity of Capparis zeylanica root extracts. Indian J. Nat. Prod. 2004; 20(1):36-39.

14. Nanjo F, Goto K, Seto R, Suzuki M, Sakai M, Hara Y. Scavenging effects of tea catechins and their derivatives on 1,1-diphenyl -2-picryl hydrazyl radical. Free Radic Biol Med. 1996;21:895-902

Source of Support: Nil, Conflict of Interest: None declared 